Tôhoku Math. Journ.

$32(1980)$, 363-373.

\title{
SPECTRAL LITTLEWOOD-PALEY DECOMPOSITIONS
}

\author{
GARTH I. GAUDRY
}

(Received April 24, 1979)

1. Introduction. Let $G$ be a compact abelian group with dual $\hat{G}$, and suppose that $E$ is a subset of $\hat{G}$. Suppose that $\left(\Delta_{j}\right)_{0}^{\infty}$ is a decomposition of $E$, i.e., that each $\Delta_{j}$ is a subset of $E$, the $\Delta_{j}$ are pairwise disjoint, and that $\bigcup \Delta_{j}=E$. We say that $\left(\Delta_{j}\right)$ is a Littlewood-Paley (or LP) decomposition of $E$ if, for every $p$ in $(1, \infty)$ there is a pair of positive constants $A_{p}$ and $B_{p}$ such that

$$
A_{p}\|f\|_{p} \leqq\left\|\left(\sum\left|S_{\Delta_{j}} f\right|^{2}\right)^{1 / 2}\right\|_{p} \leqq B_{p}\|f\|_{p}
$$

for all trigonometric polynomials $f$ with spectrum in $E$. Here $S_{\Delta_{j}} f$, which we shall frequently denote $S_{j} f$, is the partial sum of the Fourier series of $f$ over $\Delta_{j}$. The function $\left(\sum\left|S_{j}(f)\right|^{2}\right)^{1 / 2}$ is denoted $S(f)$.

When $G=\boldsymbol{T}$, and $E=\boldsymbol{Z}$, classical theorems of Littlewood and Paley furnish examples of nontrivial LP decompositions: e.g., the collection of "dyadic intervals" constitutes such a decomposition; from this basic example, many others can be built up. See [2].

Now if $\left(\Delta_{j}\right)$ is a decomposition of $E, p>2$, and each $\left(\Delta_{j}\right)$ is a singleton set, then the inequality (1) amounts to the statement that $E$ is a $\Lambda(p)$ set. In the opposite vein, if $\left(\Delta_{j}\right)$ is an LP decomposition of $E$ and $F$ is a set formed by selecting at most one element from each $\Delta_{j}$, then $F$ is a $\Lambda(p)$ set for every $p$. Given the extent of the literature on $\Lambda(p)$ sets, it seems natural to attempt to give examples of groups $\hat{G}$, proper subsets $E$ of $\widehat{G}$ and associated LP decompositions $\left(\Delta_{j}\right)$ of $E$.

As just indicated, this can be done trivially when $E$ is a $\Lambda(p)$ set for all $p$. Another way is to take an LP decomposition of a group $\hat{G}$ and then let $E$ be the union of all but one of the sets of that decomposition. Our aims should therefore be stated more precisely: we wish to produce sets $E$, and associated decompositions $\left(\Delta_{j}\right)$, such that (i) $\left(\Delta_{j}\right)$ is an LP decomposition of $E$; and (ii) $\xi_{E}$, the characteristic function of $E$, is a (Fourier) multiplier of $L^{p}$ for no $p$ other than 2. Note that if $\left(\delta_{j}\right)$ is an LP decomposition of $\hat{G}$, then the characteristic function of each $\delta_{j}$ is a Fourier multiplier of $L^{p}(1<p<\infty)$.

In her paper [1], Bonami showed how to construct various classes of sets which are $\Lambda(p)$ for all $p$, and gave precise asymptotic estimates 
for the $\Lambda(p)$ constants of these sets as $p \rightarrow+\infty$. In some instances even exact values of the constants were computed. Bonami's arguments are substantially combinatorial. By contrast, our proofs combine certain simple functional analytic ideas with ideas from Littlewood-Paley theory to produce the desired "spectral" forms of the Littlewood-Paley theorem. As pointed out in the preceding paragraph, the corresponding results of Bonami about $\Lambda(p)$ sets follow as corollaries.

2. Notation and terminology. For the most part, we follow [2] in our notation and terminology. We shall work principally with the groups $G=\boldsymbol{T}, \boldsymbol{D}_{s}=\Pi_{j=1}^{\infty}\{0,1, \cdots, s-1\}_{j}$, the product of countably many copies of the additive cyclic group of $s \geqq 2$ elements, and $\boldsymbol{T}^{\infty}$, the countablyinfinite-dimensional torus.

In $\hat{\boldsymbol{D}}_{s}$ we denote by $\boldsymbol{e}_{j}$ the element which has 1 in the $j$-th position and 0 for all other coordinates. The corresponding object in $\hat{\boldsymbol{T}}^{\infty}=\sum \boldsymbol{Z}$ is denoted $\boldsymbol{\varepsilon}_{j}$. The set $\hat{\boldsymbol{D}}_{\boldsymbol{s}}$ is canonically identifiable with a subset of $\sum \boldsymbol{Z}$ via the mapping $\sum \alpha_{j} \boldsymbol{e}_{j} \rightarrow \sum \alpha_{j} \boldsymbol{\varepsilon}_{j}$. Subsets of $\hat{\boldsymbol{D}}_{\boldsymbol{s}}$ will frequently be thought of as being transferred across into $\sum \boldsymbol{Z}$ under this same mapping.

If $x \in G$, and $\gamma \in \hat{G}$, we denote by $(x, \gamma)$ the value of $\gamma$ at $x$. In particular, if $\omega=\left(\omega_{1}, \omega_{2}, \cdots\right) \in D_{s}$, and $\sum \alpha_{n} \boldsymbol{e}_{n} \in \hat{\boldsymbol{D}}_{s}$, then $\left(\omega, \sum \alpha_{n} \boldsymbol{e}_{n}\right)$ is equal to $\exp \left((2 \pi i / s) \sum \omega_{n} \alpha_{n}\right)$. We shall customarily shorten this notation by writing $\alpha$ for the sequence $\left(\alpha_{1}, \alpha_{2}, \cdots\right)$ and $(\omega, \alpha)$ for the corresponding character value. Note that if $\alpha \in \sum\{0,1, \cdots, s-1\}$, then $\alpha$ can be thought of either as a character on $\boldsymbol{D}_{s}$ or as a character on $\boldsymbol{T}^{\infty}$. So if $\alpha$ is such a sequence, we shall denote by $(x, \alpha)$ the value of the corresponding character of $\boldsymbol{T}^{\infty}$ at the point $x \in \boldsymbol{T}^{\infty}$, and by $(\omega, \alpha)$ the value of the character of $D_{s}$ at $\omega \in D_{s}$.

If $f$ is a function on $G$, the spectrum of $f$, denoted $\operatorname{sp} f$, is the set of characters $\gamma$ at which $\hat{f}(\gamma) \neq 0$. When $E \cong \widehat{G}, T_{E}$ denotes the set of trigonometric polynomials having spectrum in $E$, and $L_{E}^{p}$ denotes the set of functions in $L^{p}$ with spectrum in $E$.

If $E \subseteq \Gamma$, and $p>2$, the $\Lambda(p)$ constant of $E$ is the number sup $\|f\|_{p} /\|f\|_{2}$, the supremum being taken over all trigonometric polynomials with spectrum in $E$.

The space $L^{p}\left(G ; l^{2}\right)$ is the space of sequences $\left(f_{j}\right)$ of measurable functions on $G$ for which

$$
\left\|\left(f_{j}\right)\right\|_{p}=\left(\int\left(\sum\left|f_{j}(x)\right|^{2}\right)^{p / 2} d x\right)^{1 / p}<\infty .
$$

Notice that to say that $\left(\Delta_{j}\right)$ is an LP decomposition of the set $E$ is a 
statement that, for each $p(1<p<\infty)$, the space $L_{E}^{p}$ is identifiable with the subspace of $L^{p}\left(G ; l^{2}\right)$ consisting of sequences $\left(f_{j}\right)$ with $\operatorname{sp} f_{j} \cong \Delta_{j}$.

3. The transfer principles. The following two lemmas will permit us to transfer classical Littlewood-Paley theorems from the group $\boldsymbol{D}_{s}$ to the group $T^{\infty}$. The first lemma is due to Bonami [1, Théorème 1, Ch. 1]; we include a proof for completeness sake.

Lemma 1. Let $\omega$ be a point in the group $\boldsymbol{D}_{s}$. Then there is a measure $\mu_{\omega}$ on $\boldsymbol{T}^{\infty}$, of total mass at most 1 , such that

$$
\hat{\mu}_{\omega}\left(\sum \alpha_{n} \varepsilon_{n}\right)=\left(\omega, \sum \alpha_{n} \boldsymbol{e}_{n}\right)
$$

for all points $\sum \alpha_{n} \boldsymbol{\varepsilon}_{n}$ of $\sum\{0,1, \cdots, s-1\} \subseteq \sum \boldsymbol{Z}=\hat{\boldsymbol{T}}^{\infty}$.

Proof (Bonami). This is because the sequence $\left\{\left(\omega, \boldsymbol{e}_{n}\right)\right\}_{n=1}^{\infty}$ is a point of $\boldsymbol{T}^{\infty}$; therefore, if $g$ is a trigonometric polynomial on $\boldsymbol{T}^{\infty}$ with spectrum in $\sum\{0,1, \cdots, s-1\}$, then $\sum \hat{g}\left(\sum \alpha_{n} \varepsilon_{n}\right)\left(\omega, \sum \alpha_{n} \boldsymbol{e}_{n}\right)$ is the value of $g$ at a point of $\boldsymbol{T}^{\infty}$. So the mapping $g \rightarrow \sum \hat{g}\left(\sum \alpha_{n} \boldsymbol{\varepsilon}_{n}\right)\left(\omega, \sum \alpha_{n} \boldsymbol{e}_{n}\right)$ is a continuous linear functional, for the sup-norm, of norm at most 1 . The result follows from the Hahn-Banach and Riesz representation theorems.

LEMma 2. Let $\left(\Delta_{j}\right)$ be a decomposition of $\hat{\boldsymbol{D}}_{s}$, and $\left(\delta_{j}\right)$ the decomposition of $I=\sum\{0,1, \cdots, s-1\} \subseteq \sum \boldsymbol{Z}$ obtained by transferring each $\Delta_{j}$ canonically into $\sum \boldsymbol{Z}$. Suppose that $1 \leqq p \leqq \infty, \omega \in \boldsymbol{D}_{s}$, and $\left(h_{j}\right) \in$ $L^{p}\left(\boldsymbol{T}^{\infty} ; l^{2}\right)$ with $\operatorname{sp} h_{j} \leqq \delta_{j}$ for every $j$. Define, for $j=0,1,2, \cdots$,

$$
h_{j}^{\omega}(x)=\sum_{\alpha \in I} \hat{h}_{j}(\alpha)(\omega, \alpha)(x, \alpha) .
$$

Then $\left(h_{j}^{\omega}\right) \in L^{p}\left(\boldsymbol{T}^{\infty} ; l^{2}\right)$, and $\left\|\left(h_{j}^{\omega}\right)\right\| \leqq\left\|\left(h_{j}\right)\right\|$.

Proof. This is simply the statement that the measure $\mu_{\omega}$ convolves $l^{2}$-valued $L^{p}$ functions into objects of the same kind, without increase of norm. The proof is much the same as the proof of the corresponding statement about convolution with scalar-valued $L^{p}$ functions.

\section{Spectral LP theorems for $\sum Z$.}

THEOREM 1. Let $s$ be an integer, $s \geqq 2,\left(\Delta_{j}\right)$ an LP decomposition of $\hat{\boldsymbol{D}}_{s}$, and $\left(\hat{\delta}_{j}\right)$ the canonical image decomposition of $I=\sum\{0,1, \cdots, s-1\}$ in $\sum \boldsymbol{Z}$. Then $\left(\delta_{j}\right)$ is an LP decomposition of $I$.

PRoof. Thanks to Lemma 1 , if $g \in T_{I}$, and $1<p<\infty$, then

$$
\int_{D_{s}} \int_{T^{\infty}}\left|\sum_{\alpha} \hat{g}(\alpha)(x, \alpha)(\omega, \alpha)\right|^{p} d x d \omega \leqq\|g\|_{p}^{p},
$$

which is to say that 


$$
\int_{T^{\infty}} \int_{D_{s}}\left|\sum \hat{g}(\alpha)(x, \alpha)(\omega, \alpha)\right|^{p} d \omega d x \leqq\|g\|_{p}^{p} .
$$

Since $\left(\Delta_{j}\right)$ is an LP decomposition of $\hat{\boldsymbol{D}}_{s}$, it follows from (1) that there is a number $B_{p}>0$ such that

$$
\int_{T^{\infty}} \int_{\boldsymbol{D}_{k}}\left(\sum_{j}\left|\sum_{\alpha \in \Delta_{i}} \hat{g}(\alpha),(\omega, \alpha)(x, \alpha)\right|^{2}\right)^{p / 2} d \omega d x \leqq B_{p}^{p}\|g\|_{p}^{p} .
$$

So, for at least one $\omega$,

$$
\int_{T^{\infty}}\left(\sum_{j}\left|\sum_{\alpha \in \Delta_{j}} \hat{g}(\alpha)(x, \alpha)(\omega, \alpha)\right|^{2}\right)^{p / 2} d x \leqq B_{p}^{p}\|g\|_{p}^{p} .
$$

But, by applying Lemma 2 to the measure $\mu_{-\omega}$, we then deduce that

$$
\int_{T_{\infty}}\left(\sum_{j}\left|\sum_{\alpha \in \Delta_{j}} \hat{g}(\alpha)(x, \alpha)\right|^{2}\right)^{p / 2} d x \leqq B_{p}^{p}\|g\|_{p}^{p} ;
$$

that is, the mapping $g \rightarrow\left(S_{\delta_{j}} g\right)$ is continuous from $L_{I}^{p}$ into $L^{p}\left(\boldsymbol{T}^{\infty} ; l^{2}\right)$. This is one half of the statement to be proved.

To prove that the mapping is onto, and so complete the proof, assume that $\left(\phi_{j}\right) \in L^{p}\left(\boldsymbol{T}^{\infty} ; l^{2}\right)$ and that $\operatorname{sp} \phi_{j} \subseteq \delta_{j}$ for each $j$. Then for every $\omega \in \boldsymbol{D}_{s},\left(\phi_{j}^{\omega}\right) \in L^{p}\left(\boldsymbol{T}^{\infty} ; l^{2}\right)$, with no increase in norm (Lemma 2). So

$$
\int_{D_{s}} \int_{T^{\infty}}\left(\left.\sum \phi_{j}^{\omega}\right|^{2}\right)^{p / 2} d x d \omega \leqq\left\|\left(\phi_{j}\right)\right\|^{p} .
$$

But, since $\left(\Delta_{j}\right)$ is an LP decomposition of $\boldsymbol{D}_{s}$, there is a constant $A_{p}$ such that

$$
\int_{T^{\infty}} \int_{D_{s}}\left(\sum\left|\phi_{j}^{\omega}\right|^{2}\right)^{p / 2} d \omega d x \geqq A_{p}^{p} \int_{T^{\infty}} \int_{D_{s}}\left|\sum_{j} \sum_{\alpha \in \dot{o}_{j}} \phi_{j}(\alpha)(\omega, \alpha)(x, \alpha)\right|^{p} d \omega d x .
$$

Reversing the order of the integrations on the right of (3), and combining with (2), we conclude that, for at least one $\omega$,

$$
A_{p}^{p} \int_{T^{\infty}}\left|\sum_{j} \sum_{\alpha \in \delta_{j}} \hat{\phi}_{j}(\alpha)(\omega, \alpha)(x, \alpha)\right|^{p} d x \leqq\left\|\left(\phi_{j}\right)\right\|_{p}^{p}
$$

Apply Lemma 2 to (4) (using $\mu_{-\omega}$ ), and the inequality

$$
A_{p}\left\|\sum_{j} \sum_{\alpha \in \dot{o}_{j}} \hat{\phi}_{j}(\alpha)(x, \alpha)\right\|_{p} \leqq\left\|\left(\phi_{j}\right)\right\|_{p}
$$

emerges.

COROLLARY 1. If $s \geqq 2$, then the following is an LP decomposition of the set $I=\sum\{0,1, \cdots, s-1\}$ in $\sum Z$ :

$$
\begin{aligned}
& \delta_{0}=\{0\}, \\
& \delta_{j}=\left\{n \in I: n_{j} \neq 0, n_{i}=0 \text { for all } i>j\right\} \quad(j \geqq 1) .
\end{aligned}
$$


Proof. This is the canonical image of the standard "corona" LP decomposition of $\hat{\boldsymbol{D}}_{\boldsymbol{s}}$ ([2, Theorem 5.4.2]).

COROLlaRY 2. If $s \geqq 2$, then the following is an LP decomposition of $I=\sum\{0,1, \cdots, s-1\}$ in $\sum Z$ :

$$
\begin{aligned}
& \delta_{0}=\{0\}, \\
& \delta_{j}=\left\{n \in I: n_{i}=0 \text { if } i>k, n_{k}=r\right\}
\end{aligned}
$$

when $j$ is of the form $j=(k-1)(s-1)+r,(k=1,2, \cdots ; r=1, \cdots$, $s-1)$.

Proof. This is the canonical image of the "coset" LP decomposition of $\hat{\boldsymbol{D}}_{\boldsymbol{s}}$ given in [5].

At the cost of notational complication, the proof of Theorem 1 can be mimicked to establish the following more general results.

TheOREM 2. Let $s$ be an integer, $s \geqq 2,\left(m_{j}\right)$ a sequence of integers, and $J=\sum_{j}\left[m_{j}, m_{j}+s-1\right] \cong \sum \boldsymbol{Z}$. For each element $\left(n_{j}\right)$ in $J$, write its entries $n_{j}$ in the form $n_{j}=k_{j} s+r_{j}$, where $k_{j} \in Z, r_{j} \in Z$, and $0 \leqq r_{j} \leqq$ $s-1$. The mapping $\tau:\left(n_{j}\right) \rightarrow\left(r_{j}\right)$ identifies $J$ with $\hat{\boldsymbol{D}}_{s}$. If $\left(\Delta_{j}\right)$ is an LP decomposition of $\hat{\boldsymbol{D}}_{s}$, then $\left(\hat{\delta}_{j}\right)=\left(\tau^{-1}\left(\Delta_{j}\right)\right)$ is an LP decomposition of $J$.

THEOREM 3. Let $s=\left(s_{j}\right)$ be a sequence of integers, each greater than 1. Denote by $\boldsymbol{D}_{s}$ the group $\Pi_{1}^{\infty} \boldsymbol{Z}\left(s_{j}\right)$, direct product of the cyclic groups $\boldsymbol{Z}\left(s_{j}\right)$, and by $I_{s}$ the subset $\sum_{j}\left\{0,1, \cdots, s_{j}-1\right\}$ of $\sum \boldsymbol{Z}$. Then $I_{s}$ is canonically identiable with $\hat{\boldsymbol{D}}_{\boldsymbol{s}}$. If $\left(\Delta_{j}\right)$ is an LP decomposition of $\hat{\boldsymbol{D}}_{\boldsymbol{s}}$, and $\left(\Delta_{j}\right)$ is canonically identified with $\left(\delta_{j}\right)$, then $\left(\delta_{j}\right)$ is an LP decomposition of $I_{s}$.

COROLLARY 3. The following family of sets is an LP decomposition of $I_{s}$ :

$$
\begin{aligned}
& \delta_{0}=\{0\}, \\
& \delta_{j}=\left\{n \in I_{s}: n_{j} \neq 0, n_{i}=0 \text { for all } i>j\right\} \quad(j \geqq 1) .
\end{aligned}
$$

Proof. Again, this is the canonical image of a corona LP decomposition ([2, Theorem 5.4.2]).

The interest in Theorems 1-3 is due to the fact that the characteristic function of the set $I$ (resp. $\left.J, I_{s}\right)$ is a Fourier multiplier of $L^{p}\left(\boldsymbol{T}^{\infty}\right)$ only when $p=2$. We shall establish this for the set $I$ only; the other proofs are similar.

Proposition 1. Let s be an integer, $s \geqq 2$, and $I=\sum\{0,1, \cdots, s-1\} \subseteq$ $\sum \boldsymbol{Z}$. Then $\xi_{I} \in M_{p}\left(\sum Z\right)$ only if $p=2$. 
Proof. By [1, Théorème 2, Ch. III], it suffices to prove that the norm of the function $1+e^{i x}+\cdots+e^{i(s-1) x}$ as a convolution operator on $L^{p}(\boldsymbol{T})$ exceeds 1 if $p \neq 2$. To establish this, fix $p>2$, and a positive integer $k$. Consider the projection of the trigonometric polynomial

$$
f=u e^{-i x}+1-k u e^{i x}
$$

onto

$$
g=1-k u e^{i x} .
$$

Here $u$ is a positive parameter which goes to 0 eventually. We claim that, by a suitable choice of $k$, determined by $p$, we can ensure that, for all sufficiently small $u$,

$$
\int\left|1-k u e^{i x}\right|^{p} d x>\int\left|u e^{-i x}+1-k u e^{i x}\right|^{p} d x,
$$

i.e.,

(5) $\quad \int\left[1+k^{2} u^{2}-2 k u \cos x\right]^{r} d x$

$$
>\int\left[1+\left(k^{2}+1\right) u^{2}-2 k u^{2} \cos 2 x-2(k-1) u \cos x\right]^{r} d x,
$$

$r$ denoting $p / 2>1$.

Now as $u \rightarrow 0$, the left side of (5) is, by Taylor's theorem, the fact that $\int \cos x d x=0$, and $\int \cos ^{2} x d x=1 / 2$, equal to

$$
\begin{gathered}
\left(1+k^{2} u^{2}\right)^{r}+\frac{r(r-1)}{2}\left(1+k^{2} u^{2}\right)^{r-2} 4 k^{2} u^{2} \cdot \frac{1}{2}+o\left(u^{2}\right) \\
=\left(1+r^{2} k^{2} u^{2}\right)+o\left(u^{2}\right) .
\end{gathered}
$$

The right side is, by the same token (use also the fact that $\int \cos 2 x d x=0$ )

$$
\begin{aligned}
\int\{1+ & \left.\left(k^{2}+1\right) u^{2}-2 k u^{2} \cos 2 x-2(k-1) u \cos x\right\}^{r} \\
= & \int\left\{1+\left(k^{2}+1\right) u^{2}\right\}^{r}+\frac{r(r-1)}{2}\left\{1+\left(k^{2}+1\right) u^{2}\right\}^{r-2}\left\{2 k u^{2} \cos 2 x\right. \\
& +2(k-1) u \cos x\}^{2}+o\left(u^{2}\right) \\
= & 1+r\left(k^{2}+1\right) u^{2}+r(r-1)(k-1)^{2} u^{2}+o\left(u^{2}\right) \\
= & 1+\left(r^{2} k^{2}-2 r^{2} k+r^{2}+2 r k\right) u^{2}+o\left(u^{2}\right) .
\end{aligned}
$$

Comparing (6) and (7), we see that we have to arrange that $r>2 k /(2 k-1)$ 
in order to have the projection mapping of norm greater than 1 . The integer $k$ can be so chosen at the outset.

CoRollaRy 4. If $E \subseteq Z$, and, for some integer $n, n \notin E$, but $n+1$, $n+2 \in E$, then $\left\|\xi_{E}\right\|_{M_{p}}>1$ for all $p \neq 2$.

5. Spectral LP theorems for $Z$. We present here an instance of an LP decomposition of a set $F$ in $Z$.

THEOREM 4. Let $\left(t_{k}\right)_{1}^{\infty}$ be a sequence of positive integers such that (i) $t_{k+1} \geqq 3 t_{k}$ for all $k$; and (ii) $\sum\left(t_{k} / t_{k+1}\right)<\infty$. Denote by $F$ the set $\left\{\sum_{k=1}^{\infty} \alpha_{k} t_{k}: \alpha_{k}=0\right.$ or $\left.1, \sum \alpha_{k}<\infty\right\} ;$ let $F_{0}=\{0\}$ and, for each $j \geqq 1$, write

$$
F_{j}=\left\{n \in Z: n=t_{j}+\sum_{k=1}^{j-1} a_{k} t_{k} ; \alpha_{k}=\begin{array}{lll}
0 & \text { or } & 1
\end{array}\right\} .
$$

Then $\left(F_{j}\right)_{0}^{\infty}$ is an LP decomposition of $F$, but $\xi_{F} \notin M_{p}$ except when $p=2$.

Proof. Denote by $E, E^{\prime}$ and $F^{\prime}$ the following sets.

$$
\begin{aligned}
& E=\left\{\alpha=\left(\alpha_{k}\right) \in \sum Z: \alpha_{k}=0 \text { or } 1\right\}, \\
& E^{\prime}=\left\{\alpha=\left(\alpha_{k}\right) \in \sum Z:\left|\alpha_{k}\right| \leqq 1\right\}, \\
& F^{\prime}=\left\{n=\sum_{k=1}^{\infty} \alpha_{k} t_{k}: \alpha \in E^{\prime}\right\},
\end{aligned}
$$

Thanks to the hypothesis (i), the sets $E^{\prime}$ and $F^{\prime}$ are canonically identifiable, as are $E$ and $F$. According to a result of Meyer [4, p. 563], if $f \in L_{F}^{p}$, say,

$$
f(x)=\sum \hat{f}\left(\sum \alpha_{k} t_{k}\right) e^{i\left(\Sigma \alpha_{k} t_{k}\right) x},
$$

and

$$
g(\omega)=\sum \hat{f}\left(\sum \alpha_{k} t_{k}\right)(\omega, \alpha)
$$

is the canonical image function on $T^{\infty}$ with spectrum in $E^{\prime}$, then $\|f\|_{p} \cong$ $\|g\|_{p},(1<p<\infty)$. But, by an argument like that in [2, 1.2.8], $\left(F_{j}\right)$ is an LP decomposition for $F$ if and only if every 0,1 -valued function on $F$ which is constant on each $F_{j}$ multiplies (in the Fourier multiplier sense) $L_{F}^{p}$ into itself. A similar statement holds for $E$ and its decomposition $\left(E_{j}\right)$ given in Corollary 1 . Since $\left(E_{j}\right)$ is an LP decomposition for $E$, it follows from Meyer's theorem that $\left(F_{j}\right)$ is an LP decomposition for $F$.

To see that $\xi_{F} \notin M_{p}$ if $p \neq 2$, recall that the proof of Proposition 1 shows that $\xi_{E}$ does not mutiply $L_{E}^{p}$ into itself if $p \neq 2$. So the proof is completed by a further appeal to Meyer's theorem. 
6. Construction of $A(q)$ sets. By using the results of the previous sections on spectral LP decompositions, we now show how to construct certain sets which are $\Lambda(p)$ for all $p>2$. Most of the results are due originally to Bonami.

THeOREM 5. Let $k$ be an integer, $k \geqq 1$. Denote by $U_{k}$ the subset of $\sum \boldsymbol{Z}$ comprising those $n=\left(n_{1}, n_{2}, \cdots\right)$ for which $n_{i} \geqq 0$ and $\sum n_{i}=k$. Then $U_{k}$ is a $\Lambda(p)$ set for all $p>2$.

Proof. We proceed by induction. When $k=1, U_{1}$ is even a Sidon set hence a $\Lambda(p)$ set.

Suppose that $1 \leqq k$ and that $U_{k}$ is known to be a $\Lambda(p)$ set. Then $U_{k+1}$ can be written as a finite union $E_{1} \cup \cdots \cup E_{k+1}$, where $E_{j}$ comprises those elements of $U_{k+1}$ whose last nonzero entry is $j$. It will be enough to prove that each $E_{j}$ is a $\Lambda(p)$ set.

Let $\left(\delta_{i}\right)_{0}^{\infty}$ be the LP decomposition of $I=\sum\{0,1, \cdots, k+1\}$ described in Corollary 1. If $f \in L_{E_{j}}^{p}\left(\boldsymbol{T}^{\infty}\right)$, then $f \in L_{I}^{p}$ and so, by Corollary 1,

$$
\|f\|_{p} \leqq A_{p}^{-1}\|S(f)\|_{p}=A_{p}^{-1}\left\|\left(\sum_{i=0}^{\infty}\left|S_{\delta_{i} \cap E_{j}} f\right|^{2}\right)^{1 / 2}\right\|_{p},
$$

$A_{p}$ being the left-hand constant in the LP inequalities for $I$. It follows from Minkowski's inequality for $p / 2>1$ that

$$
\|f\|_{p} \leqq A_{p}^{-1}\left(\sum_{i}\left\|S_{\delta_{i} \cap E_{j}} f\right\|_{p}^{2}\right)^{1 / 2} .
$$

On the other hand, $\delta_{i} \cap E_{j}$ is the translate, by an amount $\chi_{i j}$, say, of a subset of $U_{k+1-j}$, so that

$$
S_{\delta_{i} \cap E_{j}} f=\chi_{i j} f_{i},
$$

say, $f_{i}$ being supported in $U_{k+1-j}$. By the inductive hypothesis, $U_{k+1-j}$ is a $\Lambda(p)$ set, with constant $M_{j}(p)$ say. So, from (9),

$$
\left\|S_{\delta_{i} \cap E_{j}} f\right\|_{p}=\left\|f_{i}\right\|_{p} \leqq M_{j}(p)\left\|f_{i}\right\|_{2}=M_{j}(p)\left\|S_{\delta_{i}} f\right\|_{2} .
$$

Combining (8) and (10), we deduce that

$$
\|f\|_{p} \leqq A_{p}^{-1} M_{j}(p)\left(\sum_{i}\left\|S_{\delta_{i}} f\right\|_{2}^{2}\right)^{1 / 2}=A_{p}^{-1} M_{j}(p)\|f\|_{2}
$$

because the sets $\left(\delta_{i}\right)$ form a decomposition of $I$, and $f \in L_{I}^{p}$.

The proofs of the next two results, which follow the lines of the proof of Theorem 5, will be omitted.

THEOREM 6. (a) Let $k$ be a positive integer. Then $\Gamma_{k}=\left\{\left(\alpha_{i}\right) \in\right.$ $\left.\sum \boldsymbol{Z}(2): \sum \alpha_{i}=k\right\}$ is a $\Lambda(p)$ set for all $p>2$.

(b) Let $\Gamma_{k}=\left\{\boldsymbol{n}=\left(n_{i}\right) \in \sum Z: n_{i} \in\{-1,0,1\}\right.$, and $\left.\sum\left|n_{i}\right|=k\right\}$. Then 
$\Gamma_{k}$ is a $\Lambda(p)$ set for all $p>2$.

The last theorem emphasizes the point that in the results of the kind we are discussing it is not any arithmetic relationship among the coordinates that makes the set of points a $\Lambda(p)$ set. It is rather the number of nonzero entries that are permitted to appear. Theorem 7 supersedes Theorem 5 .

THEOREM 7. Let $\left(F_{j}\right)$ be a sequence of finite sets of nonnegative integers, each containing 0 , and each having $r$ elements. Let $t$ be a positive integer, and write

$$
V_{t}=\left\{n \in \sum F_{i} \text { : precisely } t \text { coordinates of } n \text { are nonzero }\right\} .
$$

Then $V_{t}$ is a $\Lambda(p)$ set for all $p>2$.

PRoof. We sketch the main modifications needed in the proof already given for Theorem 5 .

Let $S=\left(s_{j}\right)$ be a sequence of positive integers chosen so that $F_{j} \subseteq$ $\left[0, s_{j}-1\right]$ for each $j$. Denote by $I_{s}$ the set $\sum\left[0, s_{j}-1\right]$. Let $\left(\delta_{j}\right)$ be the LP decomposition of $I_{s}$ given by Corollary 3 .

If $t=1$, the result is clear, since $V_{1}$ is the union of $r$ independent, hence Sidon, sets. Assume that $1 \leqq k<t$, and that the result has been established for $V_{k}$. Suppose that $f \in L_{V_{k+1}}^{p}$. By Corollary 3, there is a constant $A_{p}$ such that

$$
\|f\|_{p} \leqq A_{p}^{-1}\|S(f)\|_{p}=A_{p}^{-1}\left\|\left(\sum_{i=0}^{\infty}\left|S_{\delta_{i}} f\right|^{2}\right)^{1 / 2}\right\|_{p} .
$$

So, by Minkowski's inequality $(p>2)$,

$$
\|f\|_{p} \leqq A_{p}^{-1}\left(\sum_{i}\left\|S_{\delta_{i}} f\right\|_{p}^{2}\right)^{1 / 2} .
$$

Now the spectrum of $S_{\delta_{i}} f$ consists of elements $n$ whose last nonzero coordinate occurs in the $i$-th position; since there are only $(r-1)$ possible candidates for the $i$-th position, $S_{\delta_{i}} f$ is the sum of $(r-1)$ trigonometric polynomials, each of the form $\chi_{i} f_{i}, \chi_{i}$ being a character of $\boldsymbol{T}^{\infty}$, and $f_{i}$ being a trigonometric polynomial with spectrum in $V_{k}$; the $(r-1)$ functions of the form $\chi_{i} f_{i}$ have disjoint spectra. But, by assumption, $V_{k}$ is a $\Lambda(p)$ set, with constant $M_{k}(p)$, say. Hence

$$
\left\|\chi_{i} f_{i}\right\|_{p}=\left\|f_{i}\right\|_{p} \leqq M_{k}(p)\left\|f_{i}\right\|_{2}=M_{k}(p)\left\|\chi_{i} f_{i}\right\|_{2} .
$$

We combine (12) and (13), as in Theorem 5, to show that

$$
\begin{aligned}
\|f\|_{p} & \leqq A_{p}^{-1}(r-1)^{1 / 2} M_{k}(p)\left(\sum_{i}\left\|S_{\delta_{i}} f\right\|_{2}^{2}\right)^{1 / 2} \\
& =A_{p}^{-1}(r-1)^{1 / 2} M_{k}(p)\|f\|_{2} .
\end{aligned}
$$


Remarks on THE $\Lambda(p)$-CONSTANTS. There is interest in the behavior, as $p \rightarrow \infty$, of the $\Lambda(p)$ constants of sets of the type constructed above. See [1] for a discussion of the matter. Looking back to Theorem 5, for instance, and the set $U_{k}$, it is easily seen that the $\Lambda(p)$ constant obtained in the proof, and there denoted $M_{k}(p)$, behaves, as $p \rightarrow \infty$, like $A_{p}^{-k+1}$. By tracing through the arguments in [2, Chapter 5], it can be shown that $A_{p}^{-1}=B_{p^{\prime}}$ ( $p^{\prime}$ the conjugate exponent), the right hand constant in the LP inequalities for the "corona" decomposition of $\hat{\boldsymbol{D}}_{k}$, behaves at worst like $p$ as $p \rightarrow+\infty$. So $M_{k}(p)$ behaves at worst like $p^{k}$. The reason that the constant we obtain does not exhibit the correct growth behavior as $p \rightarrow \infty$ is presumably because, when we utilize the Littlewood-Paley theorem for $\boldsymbol{D}_{k}$ and hence for $I \subseteq \sum \boldsymbol{Z}$, we are making a statement about functions whose spectra may lie well outside $U_{k}$ itself. By contrast, Bonami's arguments exploit directly the combinatorial structure of the sets $U_{k}$.

7. Further remarks on LP decompositions associated with "sum" sets. In another [3], we proved the following result.

THEOREM. Let $\left(n_{j}\right)$ be a Hadamard sequence of positive integers, and $k$ a positive integer. Denote by $E$ the set $\left\{\sum_{i=1}^{\infty} \alpha_{i} n_{j_{i}}: \alpha_{i}=0\right.$ or 1 , $\left.\sum \alpha_{i}=k\right\}$, and let $E$ be enumerated in increasing order as $\left\{m_{1}, m_{2}, \cdots\right\}$. Then the family of intervals $\left[0, m_{1}\right),\left[m_{1}, m_{2}\right), \cdots$ associated with $E$ is an LP decomposition of $[0, \infty)$.

This result effectively extends [1, Corollary 4 , Ch. II]. It raises the following question: suppose $E_{1}$ and $E_{2}$ are sets of positive integers each of which determines an LP decomposition of $[0, \infty) \subseteq \boldsymbol{Z}$ (via its associated family of consecutive intervals). Is it the case that $E_{1}+E_{2}$ also determines an LP decomposition of $[0, \infty)$ ? This is not so, as the following argument shows. We may even take $E_{2}$ to be Hadamard.

Construction. Let $\left(r_{k}\right)_{1}^{\infty}$ be a sequence of positive integers chosen so that

$$
2^{r_{k+1}}-(k+1)-2^{k+1}>2^{r_{k}}
$$

for all $k$. Define

$$
E_{1}=\bigcup_{k=1}^{\infty}\left\{2^{r_{k}}-j-2^{j}: 0 \leqq j \leqq k\right\} .
$$

Let $E_{1}=\left(2^{s}\right)_{s=1}^{\infty}$, a Hadamard set.

Then $E_{1}+E_{2}$ contains arbitrarily long arithmetic progressions:

$$
\left\{2^{r_{k}}-j: 0 \leqq j \leqq k\right\} \quad \text { (of length } k+1 \text { ) }
$$


and so it is not a $\Lambda(p)$ set for any $p$. A fortiori, the intervals determined by $E_{1}+E_{2}$ do not constitute an LP decomposition of $[0, \infty)$.

However, $E_{1}$ does determine an LP decomposition. The proof of this is essentially the same as the proof that if $H_{1}$ and $H_{2}$ are Hadamard sets, then $H_{1}+H_{2}$ determines an LP decomposition [3]. The only remark that need be made is that $E_{1}$ can be thought of as being built up from the Hadamard set $\left(2^{r}\right)_{k=1}^{\infty}$ by stepping backwards in each interval $\left(2^{r_{k-1}}, 2^{r_{k}}\right]$ in steps of $1,(1+2)-1,\left(2+2^{2}\right)-(1+2), \cdots,(j+1)+2^{j+1}-$ $\left(j+2^{j}\right)$, and stopping before passing $2^{r_{k+1}}$. Note that the sequence $\left(j+2^{j}\right)_{0}^{\infty}$ is Hadamard. (Meyer [4, pp. 559-561], has previously used a construction like the one above in a different, but related context.)

\section{REFERENCES}

[1] A. Bonami, Etude des coefficients de Fourier des fonctions de $L^{p}(G)$, Ann. Inst. Fourier (Grenoble) 20 (1970), 335-402.

[2] R. E. EDWARDS AND G. I. GAUDRY, Littlewood-Paley and multiplier theory, Ergebnisse der Math. 90 (1977), Springer-Verlag, Berlin-Göttingen-Heidelberg.

[3] G. I. GAUdRY, Littlewood-Paley theorems for sum and difference sets, Math. Proc. Camb. Phil. Soc. 83 (1978), 65-71.

[4] Y. Meyer, Endomorphismes des idéaux fermés de $L^{1}(G)$, classes de Hardy, et séries de Fourier lacunaires, Ann. Sci. Ecole Norm. Sup. (4) 1 (1968), 499-580.

[5] J. Peyrik̀ne et R. Spector, Sur les multiplicateurs radiaux de $L^{p}(G)$, pour un groupe localement compact totalement discontinu, C. R. Acad. Sci. Paris Sér. A 269 (1969), 973-974.

School of Mathematical Sciences

The Flinders University of South Australia

Bedford Park, South Australia 5042

Australia 
